\title{
Dissecção Robótica da Artéria Torácica Interna Direita por Esternotomia Mediana
}

\author{
Robotic Dissection of the Right Internal Thoracic Artery through Median Sternotomy
}

\author{
Fabio Biscegli Jatene, Paulo Manuel Pêgo-Fernandes, Ramez Anbar, Fábio Antonio Gaiotto, Marcello S. Barduco, \\ Roberto Kalil Filho \\ Instituto de Ensino e Pesquisa do Hospital Sírio-Libanês, São Paulo, SP - Brasil
}

A utilização de sistemas robóticos em cirurgia cardíaca visa à diminuição do trauma operatório. A revascularização do miocárdio totalmente endoscópica, assistida por robô DaVinci (Intuitive Surgical, Sunnyvale, Califórnia) é factível e seu aprendizado deve ser realizado em etapas. O primeiro passo é o preparo da artéria torácica interna esquerda, já por via totalmente endoscópica.

O caso apresentado propõe a dissecção da artéria torácica interna direita por esternotomia completa. Propõe um novo passo rumo ao procedimento completamente endoscópico, visando à diminuição de lesões decorrentes da curva de aprendizado.
The use of robotic systems in cardiac surgeries aims at decreasing the surgical trauma. The totally endoscopic myocardial revascularization, assisted by the DaVinci robot (Intuitive Surgical, Sunnyvale, California) is feasible and the learning process must be carried out in steps. The first step is the preparation of the left internal thoracic artery, through the totally endoscopic approach.

The case presented here proposes the dissection of the right internal thoracic artery through complete sternotomy. It proposes a new step directed at the totally endoscopic procedure, aiming at decreasing the injuries caused by the learning curve.

\section{Introdução}

O desenvolvimento de novos equipamentos e tecnologias tem contribuído para a diminuição do trauma cirúrgico na área da cirurgia cardiovascular ${ }^{1}$. A redução do tempo de internação, a menor utilização de hemoderivados e o melhor resultado estético são exemplos de importantes benefícios proporcionados pelos procedimentos minimamente invasivos.

Os procedimentos toracoscópicos em cirurgia cardiovascular começaram a ser publicados no início da década de 1990. Inicialmente eram videoassistidos sem o auxílio robótico ${ }^{2,3}$. Progressivamente, a tecnologia computadorizada foi sendo incorporada pelos cirurgiões e o auxílio robótico tornou-se realidade. De apenas um único braço mecânico controlado pela voz e que suportava apenas o vídeo, passamos à disponibilidade da telecirurgia totalmente assistida por um robô, dotado de console com visão tridimensional e quatro

\section{Palavras chave}

Robótica, procedimentos cirúrgicos cardíacos/métodos, procedimentos cirúrgicos minimamente invasivos, artéria torácica interna.

\footnotetext{
Correspondência: Fábio Biscegli Jatene •

Av. Dr. Enéas de Carvalho Aguiar, 44 - 5o andar - bloco 2 - sala 7 - Cerqueira César - 05403-000 - São Paulo, SP - Brasil

E-mail: fbjatene@cardiol.br, fabiojatene@incor.usp.br

Artigo recebido em 27/02/09; revisado recebido em 07/04/09; aceito em 21/08/09.
}

braços mecânicos (DaVinci Intuitive Surgical, Sunnyvale, Califórnia) (Figura 1).

São vários os procedimentos cardiovasculares passíveis de assistência robótica. O reparo da comunicação atrial, da valva mitral e a revascularização do miocárdio são os procedimentos com maior número de publicações ${ }^{4-6}$.

A curva de aprendizado para a utilização da assistência robótica compreende etapas ${ }^{7-10}$. O treinamento exaustivo em modelos experimentais é fundamental e marca o início da experiência da equipe ${ }^{9,10}$. A dissecção da artéria torácica interna esquerda é o primeiro passo para a realização da revascularização totalmente endoscópica assistida pelo robô ${ }^{7,8}$. Habitualmente, inicia-se diretamente com a dissecção endoscópica, entretanto, dificuldades no manuseio do equipamento podem acontecer, traduzindo-se em trauma e lesão do principal enxerto para a revascularização do miocárdio.

O presente trabalho propõe uma etapa anterior ao preparo da artéria torácica interna esquerda totalmente endoscópica. Relatamos a dissecção completa da artéria torácica interna direita, utilizando-se como a via de acesso a esternotomia mediana.

\section{Relato do caso}

Em dezembro de 2008, paciente do sexo masculino, 54 anos, portador de insuficiência coronariana crônica, com angina estável e candidato à revascularização do miocárdio. Como antecedentes, apresentava hipertensão arterial e dislipidemia. A cineangiocoronariografia revelava lesões graves no ramo interventricular anterior, ramo diagonalis 


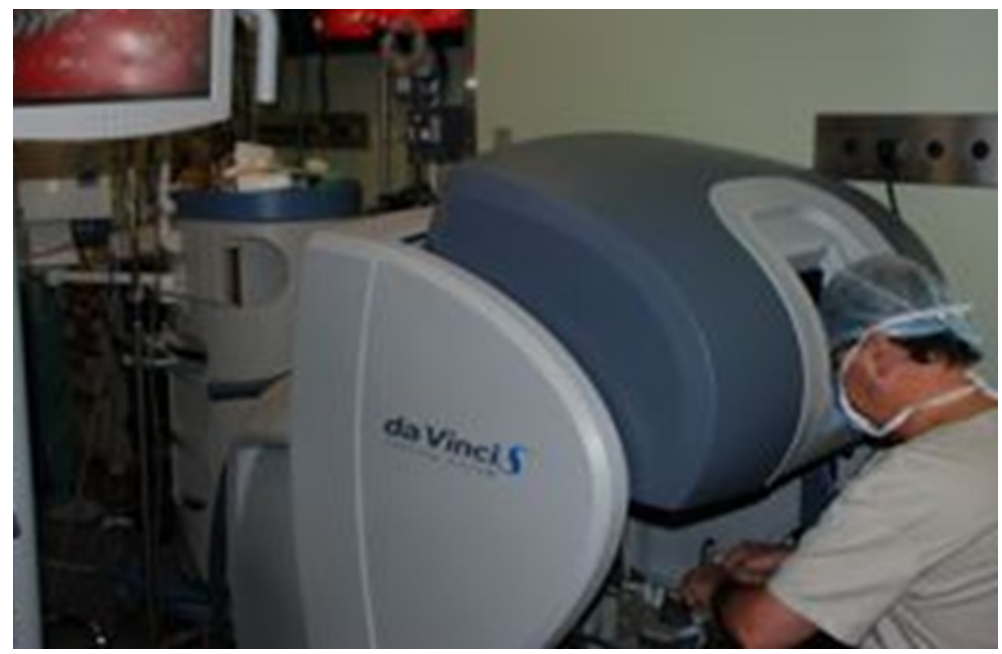

Fig. 1 - Console de operação do robô DaVinci Intuitive Surgical, Sunnyvale, Califórnia.

e ramo marginal esquerdo. A função ventricular esquerda era normal.

A operação proposta foi a revascularização do ramo interventricular anterior com a artéria torácica interna esquerda; do ramo diagonalis com a artéria torácica interna direita e ponte de safena da aorta para o ramo marginal esquerdo, em posição retroaórtica. A dissecção da artéria torácica interna direita foi realizada totalmente pelo robô

O preparo anestésico não apresentou diferença quanto à anestesia habitualmente empregada para a revascularização do miocárdio com circulação extracorpórea. A ventilação mecânica foi instituída sem a necessidade de intubação orotraqueal seletiva. Na sala operatória, o robô foi posicionado à direita do paciente, objetivando a entrada dos braços e do vídeo para o acesso à artéria torácica interna direita (Figura 2).

A esternotomia mediana foi a via de acesso utilizada. $\mathrm{O}$ preparo da veia safena magna direita, removida da coxa direita, foi através de mini-incisões. Após a abertura do saco pericárdico e exploração da cavidade, a artéria torácica interna esquerda foi preparada, de maneira esqueletizada e sem abertura pleural. Reposicionado o afastador, agora para a artéria torácica interna direita, aproximou-se o robô. A mesa operatória recebeu decúbito lateral direito de 30 graus.

Foram empregados três braços mecânicos, a saber: vídeo, eletrocautério (EndoWrist - Intuitive Surgical) e pinça de apreensão (EndoWrist deBakey - Intuitive Surgical) (Figura 2).

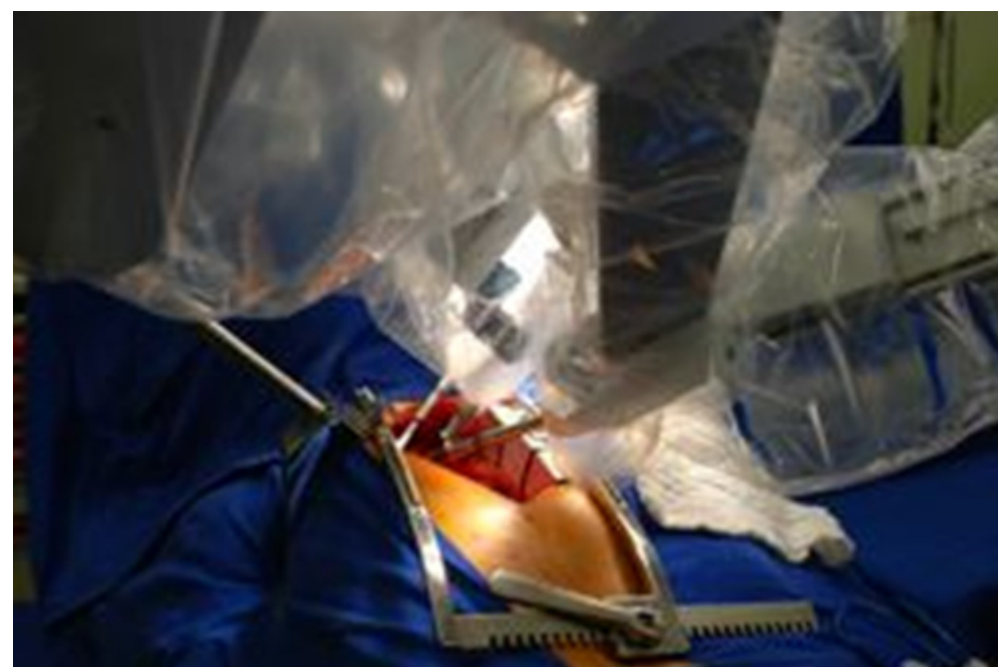

Fig. 2 - Robô em posição para o preparo da artéria torácica interna direita via esternotomia mediana. 0 robô está posicionado à direita do paciente e estão em funcionamento 3 braços: vídeo, pinça de apreensão e eletrocautério. 


\section{Relato de Caso}

O quarto braço permaneceu inativo. A dissecção foi realizada sob os mesmos princípios empregados no preparo da artéria torácica interna esquerda: esqueletizada e sem abertura da cavidade pleural. O eletrocautério (Valleylab Force II). permaneceu o tempo todo sob baixíssima voltagem.

O tempo de preparo da artéria torácica interna direita totalmente assistida com o robô e através de esternotomia foi de 50 minutos. O trecho dissecado foi desde a emergência na artéria subclávia direita até a sua bifurcação. Os ramos menores foram cauterizados e os maiores clipados sob visão direta. Ao término do preparo, não foram observados hematomas ou lesões decorrentes de tração ou pinçamento. A heparinização plena foi realizada e a artéria, cortada. O fluxo foi considerado ótimo.

A seguir, o circuito de circulação extracorpórea foi instalado. A operação transcorreu sem intercorrências, sendo realizada a revascularização previamente proposta. O tempo cirúrgico total foi de 5 horas.

A alta hospitalar foi no sétimo dia de pós-operatório, em excelentes condições clínicas.

\section{Discussão}

O equipamento utilizado (DaVinci Intuitive Surgical, Sunnyvale, Califórnia) (Figura 1) apresenta alta resolução de imagem, visualização tridimensional satisfatória, controle para diminuição de tremores e liberdade de movimentação com grande amplitude. São vantagens que favorecem e estimulam o seu emprego em procedimentos minimamente invasivos.

São vários os relatos de preparo das artérias torácicas internas para a revascularização do miocárdio, de maneira totalmente endoscópica. Entretanto, a curva de aprendizado pode ser longa e, eventualmente, lesões podem ocorrer ${ }^{7,8}$.

São vários os modelos experimentais propostos para o treinamento em cirurgia robótica ${ }^{9,10}$. Simulações em modelos não vivos introduzem o cirurgião à tecnologia robótica. Modelos experimentais em animais permitem aproximação da realidade e são fundamentais antes da operação em seres humanos. A dissecção da artéria torácica interna direita com o robô e por esternotomia mediana pode ser o primeiro passo.

Empregando-se a esternotomia mediana, o acesso dos braços do robô à artéria é facilitado, comparando-se ao tórax fechado. A possibilidade de intervenção imediata em casos de sangramento transmite segurança e tranquilidade ao cirurgião, em adaptação à técnica. Um cirurgião assistente permanece em frente aos braços do robô com facilidade para agir.

A atividade do cirurgião é idêntica àquela realizada quando do preparo totalmente endoscópico da artéria torácica interna esquerda. O campo visual é o mesmo e os movimentos dos braços também são iguais. O avanço para o preparo totalmente endoscópico torna-se natural.

O controle anestésico do paciente em nada diferiu da anestesia rotineira para a revascularização do miocárdio. Não houve necessidade de intubação orotraqueal seletiva e nem redução do volume ventilatório. O sangramento foi desprezível.

O tempo de preparo foi satisfatório, considerando-se a curva de aprendizado. Seguramente, seria maior se o preparo escolhido fosse o totalmente endoscópico.

Não foram notadas lesões decorrentes da dissecção com o robô. Estima-se em $6 \%$ as lesões na fase de aprendizado e o valor de $2 \%$ é aceitável após amplo treinamento ${ }^{7,8}$. O aprendizado empregando-se a esternotomia convencional pode diminuir estes valores, principalmente na fase inicial.

A curva de aprendizado deve ser considerada em todas as áreas cirúrgicas, principalmente quando as inovações tecnológicas são incorporadas. Acreditamos que o preparo da artéria torácica interna direita, via esternotomia mediana, pode ser um importante passo inicial. É uma maneira segura de se iniciar a cirurgia cardíaca totalmente endoscópica assistida por robô.

\section{Agradecimentos}

Aos Drs. Paulo Chap-Chap, Ricardo Abdalla e Marcelo Cerdan Torres, pelo suporte para realização deste procedimento.

\section{Potencial Conflito de Interesses}

Declaro não haver conflito de interesses pertinentes.

\section{Fontes de Financiamento}

O presente estudo não teve fontes de financiamento externas.

\section{Vinculação Acadêmica}

Não há vinculação deste estudo a programas de pósgraduação.

\section{Referências}

1. Jatene F, Gaiotto FA, Monteiro R. Cirurgia cardíaca minimamente invasiva. In Tratado de Cardiologia da SOCESP. 2a ed. São Paulo: Manole; 2008. p. 2554-60.

2. Jatene FB, Fernandes PM, Stolf NA, Kalil R, Hayata AL, Assad R, et al. Cirurgia de revascularização do miocárdio minimamente invasiva com utilização da videotoracoscopia. Arq Bras Cardiol. 1997; 68: 107-11.

3. Jatene FB, Pêgo-Fernandes PM, Hayata AL, Arbulu HE, Stolf NA, Oliveira SA, et al. VATS for complete dissection of LIMA in minimally invasive coronary artery

bypass grafting. Ann Thorac Surg. 1997; 63: 110-3.

4. Ak K, Aybek T, Wimmer-Greinecker G, Ozaslan F, Bakhtiary F, Moritz A, et al. Evolution of surgical techniques for atrial septal defect repair in adults: a 10-year single-institution experience. J Thorac Cardiovasc Surg. 2007; 134: 757-64.

5. Chitwood WR Jr, Rodriguez E, Chu MW, Hassan A, Ferguson TB, Vos PW, et al. Robotic mitral valve repairs in 300 patients: a single-center experience. J Thorac Cardiovasc Surg. 2008; 136: 436-41. 


\section{Relato de Caso}

6. Schachner T, Feuchtner GM, Bonatti J, Bonaros N, Oehlinger A, Gassner E, et al. Evaluation of robotic coronary surgery with intraoperative graft angiography and postoperative multislice computed tomography. Ann Thorac Surg. 2007; 83: 1361-7.

7. Oehlinger A, Bonaros N, Schachner T, Ruetzler E, Friedrich G, Laufer G, et al. Robotic endoscopic left internal mammary artery harvesting: what have we learned after 100 cases? Ann Thorac Surg. 2007; 83: 1030-4.
8. Bonatti J, Schachner T, Bernecker O, Chevtchik O, Bonaros N, OttH, etal. Robotic totally endoscopic coronary artery bypass: program development and learning curve issues. J Thorac Cardiovasc Surg. 2004; 127: 504-10.

9. Ishikawa N, Watanabe G, Hirano Y, Inaki N, Kawachi K, Oda M. Origami using da Vinci surgical system. Surg Endosc. 2007; 21: 1252-3.

10. Ishikawa N, Sun Y, Nifong W, Watanabe G, Chitwood WR. Thoracoscopic lobectomy with the da Vinci Surgical System. Innovations. 2006; 1: 169-70. 